\title{
REACH: Dritte Registrierungsfrist startet im Mai
}

Mit der EU-Verordnung REACH müssen Hersteller und Importeure von Chemikalien ihre Produkte in gestaffelten Fristen registrieren. Die dritte Registrierungsfrist, die im Mai 2013 startet, betrifft vor allem kleine und mittlere Unternehmen (KMU), die sich nach Aussage von TÜV Süd frühzeitig mit der Umsetzung der umfangreichen Anforderungen befassen sollten.

$\mathrm{N}$ icht unter REACH registrierte Stoffe dürfen nach Ablauf der jeweiligen Frist innerhalb der Europäischen Union nicht mehr hergestellt oder in Verkehr gebracht werden. „Aber zu vielen derzeit auf dem Markt befindlichen Chemikalien existieren noch keine genauen Informationen über das Gefahren- und Risikopotenzial“, sagt Dr. Dieter Reiml, REACH-Experte bei der TÜV SÜD Industrie Service GmbH. Zudem sei noch nicht ausreichend bekannt, dass die Verantwortung für den sicheren Umgang mit chemischen Stoffen von staatlichen Stellen weitgehend auf die Industrie übergegangen ist.

Das Ziel der 2007 in Kraft getretenen REACH-Verordnung besteht darin, Beschäftigte, Verbraucher und Umwelt noch besser zu schützen. Die Basis dafür ist eine europaweit einheitliche $\mathrm{Ge}$ setzgebung, die rund 60.000 Stoffe betrifft. Die erste Registrierungsfrist ist bereits Ende 2010 abgelaufen. Sie galt für chemische Stoffe, die in großen Mengen vermarktet werden. Bis 31. Mai 2013 müssen alle Stoffe im Mengenband zwischen 100 bis 1.000 Tonnen pro Jahr registriert werden. „Dann dürften insgesamt knapp 10.000 Stoffe erfasst sein“, erklärt Dr. Reiml. „Und Mitte 2018 endet schließlich die Frist für die verbliebenen kleineren Stoffmengen ab 1 Tonne pro Jahr.“

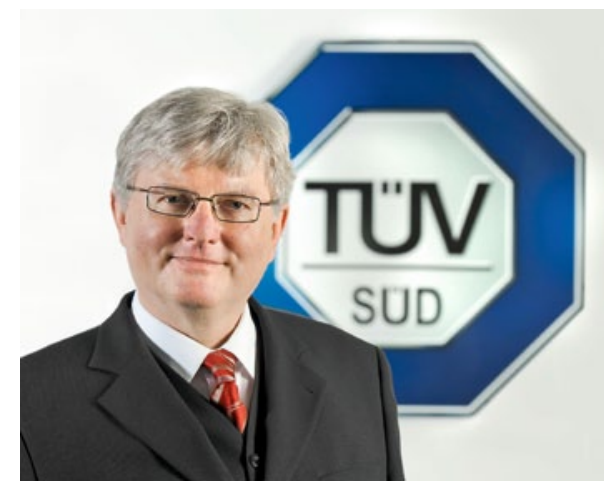

Dr. Dieter Reiml, REACH-Experte bei der TÜV Süd Industrie Service GmbH: „Die sinnvolle und vorausschauende Auslegung der Verordnung kann wettbewerbsund sogar existenzrelevant sein, wenn es beispielsweise zu Registrierungsfehlern kommt.“
KMU sind gefordert

Zu den REACH-Anforderungen zählt unter anderem, dass bei gefährlichen Stoffen und Gemischen vom Lieferanten ein Sicherheitsdatenblatt vorzulegen ist, in dem auch die Verwendung des Stoffes berücksichtigt werden muss. Darin sollen eine Sicherheitsbeurteilung und vorgeschlagene Maßnahmen zur Risikominderung enthalten sein. Wichtig ist zudem die zuverlässige Informationsweitergabe innerhalb der Lieferkette - vom Hersteller oder Importeur über den Händler bis zum Anwender.

„Weil die REACH-Anforderungen sehr umfangreich sind und wichtige Kapazitäten binden können, sollten insbesondere KMU schon heute aktiv werden“, rät Dr. Dieter Reiml. Werden bestimmte Stoffe in unteren Mengenbändern hergestellt oder importiert, ist zum einen oft nicht bekannt, ob die Mengen- schwelle von 1 Tonne pro Jahr tatsächlich überschritten wird oder der jeweilige Stoff im Jahr 2018 überhaupt noch vermarktet wird. Zum anderen verfügen nicht alle Unternehmen über ausreichend Personal, um sich mit REACH intensiv genug zu befassen.

Die sogenannte Mittelstandsinitiative von TÜV Süd sichert als spezieller REACH-Service für KMU die fristgerechte, wirtschaftliche und erfolgreiche Registrierung von chemischen Stoffen. Üblicherweise sind KMU sogenannte Co-Registranten, die sich bei der Einreichung des Registrierungsdossiers unterstützen lassen können. Damit entfällt für die Unternehmen beispielsweise die Einarbeitung in das REACH-Regelwerk wie auch in IUCLID und REACH-IT sowie die ständige Pflege dieser EDV-Systeme.

Weitere Infos: www.tuev-sued.de/is

\section{Workshop: Substitution unter REACH}

D ie Bundesanstalt für Arbeitsschutz und Arbeitsmedizin (BAuA) veranstaltet am 25. April in Dortmund den Workshop „Substitution unter REACH Zulassungsantrag oder auf Alternativen umstellen?“. Thematisiert wird die Verwendung zulassungspflichtiger Stoffe unter REACH. Betroffene Unternehmen sind grundsätzlich verpflichtet, eine Substitution zulassungspflichtiger Stoffe umzusetzen. Wenn dies aus Sicht des Unternehmens nicht möglich ist, muss es eine Zulassung beantragen, um den Stoff für eine bestimmte Zeit weiterverwenden $\mathrm{zu}$ dürfen. In der Veranstaltung werden die Beurteilungskriterien für die Substitution behandelt, die in Zusammenhang mit Voraussetzungen für die Genehmigung von Zulassungsanträgen relevant sind.

Weitere Infos: www.baua.de/termine 